\title{
BLACK TURMERIC DATABASE: A DATABASE OF NATURAL COMPOUNDS FROM CURCUMA CAESIA ROXB.
}

\author{
MUKUNTHAN KS ${ }^{1}$, BALAJI B ${ }^{2 *}$, PATEL TN ${ }^{3}$ \\ ${ }^{1}$ Department of Biotechnology, Manipal Institute of Technology, Manipal Academy of Higher Education, Manipal, Karnataka, India. \\ ${ }^{2}$ Department of School of Information Sciences, Manipal Academy of Higher Education, Manipal, Karnataka, India. ${ }^{3}$ Department of \\ Medical Biotechnology, Vellore Institute of Technology, Vellore, Tamil Nadu, India. \\ Email: balaji.b@manipal.edu
}

Received: 11 December 2017, Revised and Accepted: 17 January 2018

\section{ABSTRACT}

Objective: Most species have their trademark metabolites or a class of chemicals that are generally disseminated inside the genus variety. Secondary metabolites from the black turmeric are predominantly camphor, terpenes, lactones, alkaloids, and phenols classification. A database was constructed with the predicted absorption, distribution, metabolism, elimination, and toxicity, druglikeliness, and physiochemical properties of the exclusive black turmeric 103 compounds.

Methods: The interface has been designed using Microsoft Structured Query Language platform, keeping in mind the ease of use for a researcher. To support this, the entire set of chemicals and their properties was uploaded in database. Database is used as it is very efficient and supports multiple users at the same time.

Results: Black turmeric database gives complete data of compounds by means of 4 segments. Physiochemical, pharmacodynamics, pharmacokinetics, druglikeliness, and molecular information are displayed with chemical structure.

Conclusion: We solidly believe that the information from this database are the sources to infer novel pharmaceutical chemical compounds for medication.

Keywords: Black turmeric, Natural products, Absorption, distribution, metabolism, elimination and toxicity and Microsoft Structured Query Language.

(C) 2018 The Authors. Published by Innovare Academic Sciences Pvt Ltd. This is an open access article under the CC BY license (http://creativecommons. org/licenses/by/4. 0/) DOI: http://dx.doi.org/10.22159/ajpcr.2018.v11i3.24204

\section{INTRODUCTION}

Nature, the most productive source of chemical and biological qualities is the most encouraging repository of bioactive chemical compounds. Especially, the green source on the earth's surface offers an excellent source for exploration of novel drug molecules. Such new biologically active metabolites give material and key structures to the improvement of new items in chemical, pharmaceutical, food, cosmetic, and agrochemical sectors. Current drug research and development focuses on screening therapeutic compounds from nature for anticancer, antifungal, antitubercular, antiparasitic, antibacterial, and antiinflammatory as well as other biological activities [1-9]. High throughput screening of plant small chemical molecules for a given drug target can be accomplished, if just the metabolites are accessible in a database [10]. Making a database of metabolite items and imparting it to drug discovery group will comprehend fundamental mechanism of the metabolites and may decrease the timeline in discovery stages of drug [11]. An openly available database that gives exhaustive data about these compounds is consequently useful to the applicable drug discovery groups [12].

Curcuma caesia (black turmeric/black zedoary), an endangering perennial herb of the family Zingiberaceae, is indigenous to India. Black turmeric is used as a spice, food preservative, and coloring material commonly in the Indian subcontinent. Black turmeric is the latest in spice family which are chemically being analyzed, with few reports published describing 100 plus secondary metabolites from this plant, and they will remain as a source for new bioactive compounds. This database is centered around biologically active compounds that aim the pharmaceutical market, alongside the physiochemical properties.
Secondary metabolites from black turmeric are predominantly camphor, terpenes, lactones, alkaloids, and phenols classification. Most species have their trademark metabolites or a class of chemicals that are generally disseminated inside the genus variety. The user interface has been designed keeping in mind the ease of the use for a researcher. The database has been designed using Microsoft Structured Query Language (SQL) platform. To support this, we have uploaded the entire set of chemicals and their properties on a database. Database is used as it is very efficient and supports multiple users at the same time. Moreover, the list of chemicals can be added timely. Previously, we have performed screening for plant metabolites from $C$. caesia. By building database, we can encourage the virtual screening process for the lead molecule identification. We decidedly assume that the constituents from this database are the sources to novel pharmaceutical compounds.

\section{METHODS}

\section{Data collection}

In this present study, 103 chemical structures of $C$. caesia collected from experiment and literature [13-20] were drawn using ACD/Chemsketch 12 Version (ACD/Labs, 2010). The chemical structures were tested for its absorption, distribution, metabolism, elimination, and toxicity (ADMET) profile and druglikeliness through PreADMET (http:// preadmet.bmdrc.org) open source tool [21]. Compounds in database are annotated by molecular property. Chemsketch (ACD/Labs, 2010) was employed to calculate physiochemical properties of the twodimensional (2D) structures. Molecular properties essential for every stage of drug development were predicted using Medchem Designer (MedChem Designer v. 2.0, Simulations Plus, Inc.: Lancaster, CA, USA). 
Database implementation

The data were stored in a Microsoft SQL server in a windows operating system. The dataflow is shown in Fig. 1.

\section{Database interface}

The database interface is implemented using ASP. This database is searchable by compound numbers from 1 to 103 . In a query, a user can specify a number in search field. No wild characters were supported in the search field.

\section{RESULTS}

The database is extraordinary in giving complete data of compounds from $C$. caesia by means of 4 noteworthy segments. The main navigation menus of the database are predicted ADMET, druglikeliness, physiochemical properties, and molecular properties. The ADMET part of the database section shows the compound's predicted adsorption for human intestinal absorption \%, human epithelial colorectal adenocarcinoma cells (Caco-2), Madin-Darby canine kidney cells, distribution for skin, plasma protein binding and blood-brain barrier, metabolism with inhibition and substrate of liver enzymes cytochrome P450 2C19, cytochrome P450 2C9, cytochrome P450 2D6, and cytochrome P450 3A4 inhibition and substrate, mutagenicity (Ames test), and toxicity (mouse and rat). The data encompass qualitative and quantitative data. Druglikeliness is then followed with compounds predicted by qualitative data such as lead like rule, rule of five, modern drug data report like rule, World Drug Index like rule, and Comprehensive Medicinal Chemistry like rule.

The physiochemical properties of chemical structure like its name in simplified molecular-input line-entry specification (SMILES), InChl followed by molecular formula, formula weight, composition, molar refractivity, molar volume, parachor, index of refraction, surface tension, density, dielectric constant, polarizability, monoisotopic mass, nominal mass, average mass, $\mathrm{M}+, \mathrm{M}-,[\mathrm{M}+\mathrm{H}]+$ $[\mathrm{M}+\mathrm{H}]-, \quad[\mathrm{M}-\mathrm{H}]+$, and $[\mathrm{M}-\mathrm{H}]-$. The pre-computed molecular properties such as $\mathrm{S}+\log \mathrm{P}, \mathrm{S}+\log \mathrm{D}, \mathrm{M} \log \mathrm{P}, \mathrm{Mwt}, \mathrm{HBDH}, \mathrm{MNO}$, topological polar surface area, and rule of five were predicted using MedChem Designer. Entering the chemical number (1-103) with the search bar and clicking on the tab will display the information about the chemical molecule with the assigned structures. The sample interface is shown in Fig. 2.

DISCUSSION

The chemical information displayed can be utilized for cheminformatics research such as pharmacophore inquiry, molecular docking, and quantitative structure-activity relationship analysis. It also depicts the array of secondary metabolites accessible in $\mathrm{C}$. caesia and the need to protect the imperiling plant species. This database will support the identification of new leads for targeting prominent disease-causing proteins. The database also implements the predicted pharmacokinetics information for the chemical features in the compounds. Until date, there is no comprehensive database for the spice. Hence, this database can be utilized as an alternative source of information for new lead candidates. The chemical structures are available from database searching to theoretical chemists for access using their computer. The variations in chemical structures and their properties differ considerably could also help scientist draw parallel conclusions. Using exact match searches, similarity searches, and substructure searches, set of compounds can be eliminated with properties that were considered to be undesirable for drug. The addition of more data on molecular interactions with advanced interactive visualizer, new structure information in protein data bank, MOL, and SMILES format, which can be downloaded for molecular visualization and addition of chemical descriptors, will be done periodically. The purpose of this study is to make the database available on the World Wide Web for free access by researchers interested in the field of cheminformatics.

\section{CONCLUSION}

If there is a large and detailed database about the drugability of the $C$. caesia compounds or natural products from different origin,

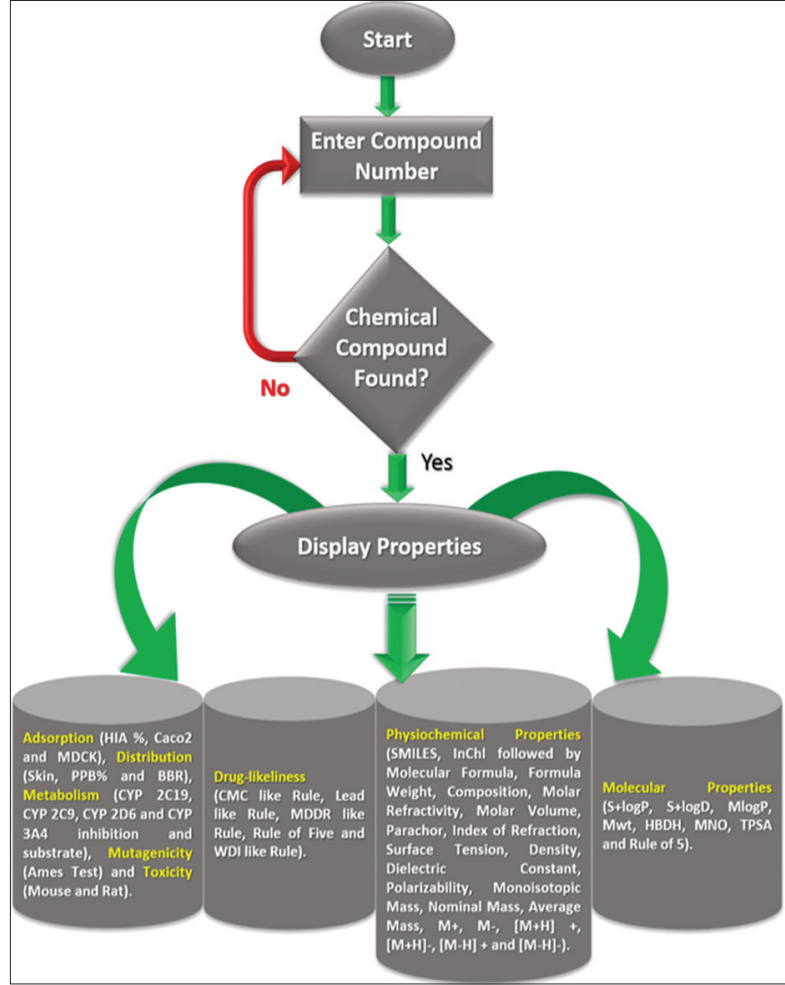

Fig. 1: Dataflow of black turmeric database

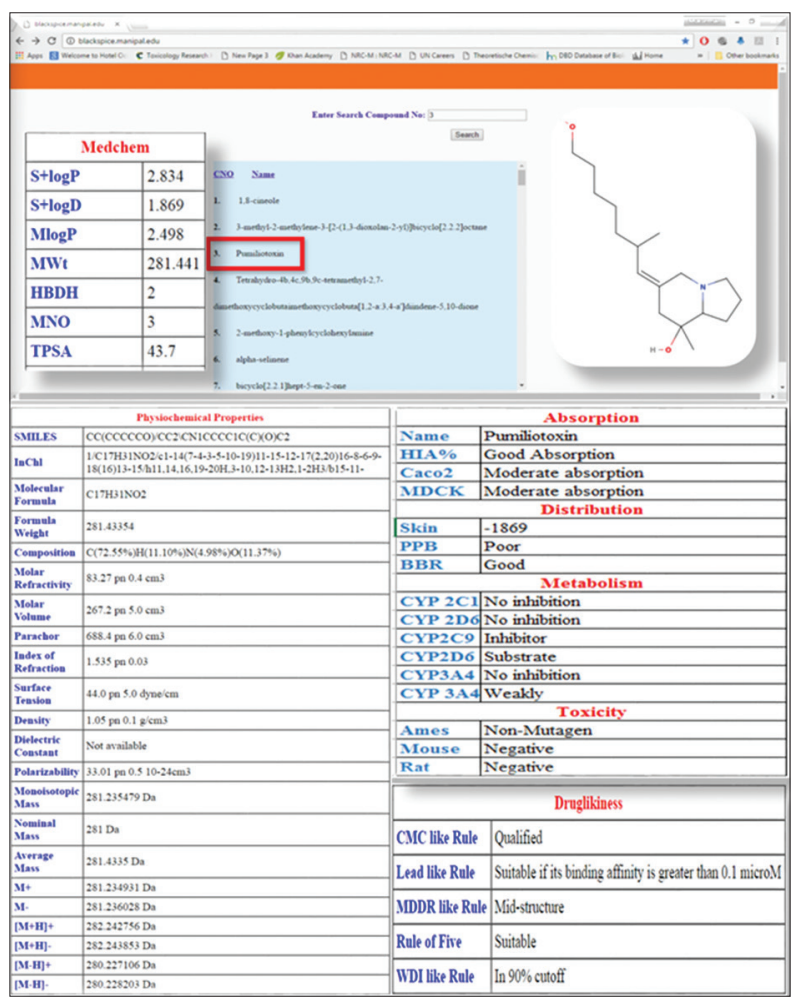

Fig. 2: Snapshot of black turmeric database

this might encourage researchers to select a more appropriate lead structure for the desired purpose. Hence, a database was developed and will be made available online, where one could quickly find the compounds from the selected spice $C$. caesia. The dataset and web interface will be updated regularly with inclusion of more information on subatomic level interaction, representation devices, and extra chemical descriptors. We also have planned to include datasets of other 
black variety crops. Black turmeric database is a platform is publicly available at http: \\blackspice.manipal.edu.

\section{ACKNOWLEDGMENTS}

The authors thank Mr. Sathish Kamath M, Asst. Director-IT Information Systems Department, Manipal University, for his support for hosting the website.

\section{CONFLICT OF INTERESTS}

We declare that we have no conflict of interest.

\section{AUTHORS CONTRIBUTION}

M.K.S. and B.B. carried out the data collection and database construction. P.T.N. encouraged M.K.S. and B.B. to investigate and supervised the project work. M.K.S. and B.B. wrote the manuscript with support from P.T.N.

\section{REFERENCES}

1. Ved P, Nishita J, Mrinal S. A review on medicinal properties of Centella asiatica. Asian J Pharm Clin Res 2017;10:69-4.

2. Prasad PN, Madakka M, Rajesh B. Pupalia Lappacea Juss [L]: A review of phytochemistry and therapeutic application. Asian J Pharm Clin Res 2014;7:15-8.

3. Shuaibu BS, Md Fadzelly AB, Maryati M, Siti FS, Md Murtala M. Ethnobotanical, phytochemical, and pharmacological properties of Nepenthes Species: A review. Asian J Pharm Clin Res 2017;10:16-9.

4. Muchtaridi M, Cindy AW. Anticancer potential of $\alpha$-mangostin. Asian J Pharm Clin Res 2017;10:440-5.

5. Lokesh R, Manasvi V, Praveena LB. Antibacterial and antioxidant activity of saponin from Abutilon Indicum Leaves. Asian J Pharm Clin Res 2016;9:344-7.

6. Priyanka K, Shivakumar BM. Chikungunya drug target database: A comprehensive database of chikungunya drug targets. Asian J Pharm Clin Res 2016;9:134-7.

7. Archana D, Dixitha M, Santhy KS. Antioxidant and anti clastogenic potential of Piper Longum L. Int J Appl Pharm 2015;7:11-4

8. Ateufack G, Mikwangock DH, Dongmo FB, Yousseu NW,
Kamanyi A. Antiulcerogenic and antiulcer activities of Dissotis thollonii (Melastomataceae) leaves in rats. Int J Pharm Pharm Sci 2015;7:62-9.

9. Sumanya H, Lavanya R, Umamaheswara RC. Evaluation of in vitro anti-oxidant and anti-arthritic activity of methanolic extract of marine green algae Caulerpa Racemosa. Int J Pharm Pharm Sci 2015;7:340-3.

10. Wasimul H, Shakti PP, Barij NS. Evaluation of taxifolin and phloretin as antiangiogenic flavonoids: An in vivo, in vitro experimental analysis. Int J Pharm Pharm Sci 2015;7:72-9.

11. Lazo JS, Brady LS, Dingledine R. Building a pharmacological lexicon: Small molecule discovery in academia. Mol Pharmacol 2007;72:1-7.

12. Davis GD, Vasanthi AH. Seaweed metabolite database (SWMD): A database of natural compounds from marine algae. Bioinformation 2011;5:361-4

13. Banerjee AK, Kaul VK, Nigam SS. Chemical examination of the essential oil of Curcuma caesia Roxb. Essenze Derivati Agrumari 1984;54:117-21.

14. Buddhasukh D, Smith J, Ternai B. Essential oil of Curcuma caesia Roxb. J Sci Fac CMU 1995;21:14-6.

15. Pandey AK, Chowdhury AR. Volatile constituents of the rhizome oil of Curcuma caesia Roxb. from central India. Flavour Frag J 2003;18 $: 463-5$.

16. Liu Y, Roy SS, Nebie RH, Zhang Y, Nair MG. Functional food quality of Curcuma caesia, Curcuma zedoaria and Curcuma aeruginosa endemic to northeastern India. Plant Foods Hum Nutr 2013;68:72-7.

17. Mukunthan KS, Kumar VA, Balaji S, Trupti NP. Analysis of essential oil constituents in rhizome of Curcuma caesia Roxb. from South India. J Essent Oil Bear Plant 2014;17:647-51.

18. Vairappan CS, Elias UM, Ramachandram TR, Kamada T. Secondary metabolites from rhizome of Curcuma caesia Roxb. (Zingiberaceae). Biochem Syst Ecol 2013;48:107-10.

19. Mukunthan KS, Amritendu B, Patel TN. Regression analysis: Identifying molecular descriptors for HIA, MDCK and Caco-2. Int J Pharm Sci Rev Res 2016;37:205-9.

20. Mukunthan KS, Satyan RS, Patel TN. Pharmacological evaluation of phytochemicals from South Indian black turmeric (Curcuma caesia Roxb.) to target cancer apoptosis. J Ethnopharmacol 2017;209:82-90.

21. Lee SK, Lee IH, Kim HJ, Chang GS, Chang JE. The PreADME Approach: Web-Based Program for Rapid Prediction of PhysicChemical, Drug Absorption and Drug-Like Properties. Boston, MA: Blackwel Publishing; 2003. p. 418-20. Available from: http://www. preadmet.bmdrc.org. 